\title{
The Effect of Repeated Virtual Nicotine Cue Exposure Therapy on the Psychophysiological Responses: A Preliminary Study
}

\author{
Jung-Seok Choi', Sumi Park', Jun-Young Lee', Hee-Yeon Jung', \\ Hae-Woo Lee ${ }^{1,2}$, Chong-Hyeon $\mathrm{Jin}^{3}$ and Do-Hyung Kang ${ }^{2}$ \\ ${ }^{1}$ Department of Psychiatry, SMG-SNU Boramae Medical Center, Seoul, Korea \\ ${ }^{2}$ Department of Neuropsychiatry, Seoul National University Hospital, Seoul, Korea \\ ${ }^{3} \mathrm{Han}$ Young Foreign Language High School, Seoul, Korea
}

\begin{abstract}
Objective Smoking related cues may elicit smoking urges and psychophysiological responses in subjects with nicotine dependence. This study aimed to investigate the effect of repeated virtual cue exposure therapy using the surround-screen based projection wall system on the psychophysiological responses in nicotine dependence.

Methods The authors developed 3-dimensional neutral and smoking-related environments using virtual reality (VR) technology. Smoking-related environment was a virtual bar, which comprised both object-related and social situation cues. Ten subjects with nicotine dependence participated in 4-week (one session per week) virtual cue exposure therapy. Psychophysiological responses [electromyography (EMG), skin conductance (SC), and heart rate] and subjective nicotine craving were acquired during each session.

Results VR nicotine cue elicited greater psychophysiological responses and subjective craving for smoking than did neutral cue, and exposure to social situation cues showed greater psychophysiological responses in SC and EMG than did object-related cues. This responsiveness decreased during the course of repeated therapy.

Conclusion The present study found that both psychophysiological responses and subjective nicotine craving were greater to nicotine cue exposure via projection wall VR system than to neutral cues and that enhanced cue reactivity decreased gradually over the course of repeated exposure therapy. These results suggest that VR cue exposure therapy combined with psychophysiological response monitoring may be an alternative treatment modality for smoking cessation, although the current findings are preliminary.
\end{abstract}

Psychiatry Investig 2011;8:155-160

Key Words Virtual reality, Nicotine dependence, Craving, Psychophysiological response.

\section{INTRODUCTION}

Cigarette smoking accounts for about $30 \%$ of all cancer deaths and is a major cause of heart disease, cerebrovascular disease, chronic bronchitis, and emphysema. ${ }^{1}$ Although the harmful effects of smoking on health are widely recognized, it has been estimated that each year fewer than $10 \%$ of smokers attempt to quit and only $3 \%$ of smokers do so successfully. ${ }^{2}$

Individuals with substance dependence are believed to work through classical conditioning in their acquisition of addictive

Received: November 14, 2010 Revised: March 5, 2011

Accepted: March 22, 2011 Available online: April 25, 2011

$\triangle$ Correspondence: Do-Hyung Kang, MD, PhD

Department of Neuropsychiatry, Seoul National University Hospital, 101 Daehak-ro, Jongno-gu, Seoul 110-799, Korea

Tel: +82-2-2072-0690, Fax: +82-2-744-7241, E-mail: basuare@paran.com

(a) This is an Open Access article distributed under the terms of the Creative Commons Attribution Non-Commercial License (http://creativecommons.org/licenses/by$\mathrm{nc} / 3.0$ ) which permits unrestricted non-commercial use, distribution, and reproduction in any medium, provided the original work is properly cited. behaviors. ${ }^{3}$ It has also been postulated that repeated exposure to drug-related cues in the absence of drug use result in extinction of drug cue reactivity. Cue exposure therapy refers to the repeated exposure to drug-related cues that are aimed at reducing the cue reactivity by extinction. Cue reactivity comprises both subjective reports of craving and patterns of psychophysiological responses such as heart rate (HR) and skin conductance (SC) in persons with addictive behaviors. ${ }^{4}$ In nicotine dependence, smokers reported greater urges to smoke in response to a wide variety of cues, including interpersonal interaction cues, ${ }^{5}$ in vivo visual and olfactory cues, ${ }^{6}$ and various contexts cues associated with a high risk of relapse. ${ }^{7}$ In addition, psychophysiological responses to smoking cues might be considered objective markers of cue reactivity. A few previous reports described psychophysiological reactions associated with smoking cue exposure. ${ }^{8-10}$ However, results from previous studies were inconsistent with regard to psychophysiological responses. Furthermore, a strong desire or craving to 
smoke seems to play an important role in the maintenance of cigarette smoking. ${ }^{11}$ Therefore, cue exposure therapy by reducing the cue reactivity has been proposed as a potential addiction treatment. ${ }^{12,13}$ However, to date, there have been only few reports about cue exposure therapy performed in subjects with nicotine dependence. ${ }^{14-16}$

Virtual reality (VR) allows an individual to become immersed in a computer-generated situation. VR technology provides immersive and interactive 3-dimensional systems that have led to its successful use in the exposure-based treatment of a variety of psychiatric disorders including acrophobia, ${ }^{17}$ fear of flight ${ }^{18}$ and post traumatic stress disorder. ${ }^{19}$ Furthermore, VR systems have also been developed for the use of addictive behaviors. Some authors have reported the usefulness of VR for cue reactivity on alcohol, ${ }^{20}$ cannabis $^{21}$ and cigarette smoking. ${ }^{14,22}$ Saladin et al. ${ }^{4}$ also suggested the application of VR to cocaine cue exposure therapy. Based on these reports, VR technology might have advantages in cue exposure therapy by increasing the salience of cues. Furthermore, to date, most studies using VR cue exposure therapy adopted the head-mounted device (HMD) system, which may have limitations such as a cybersickness during virtual exposure..$^{23}$ In addition, only few studies measured psychophysiological responses during VR cue exposure.

In this preliminary study, the authors aimed to investigate the effect of a 4-week short-term virtual cue exposure therapy on the psychophysiological responses in subjects with nicotine dependence using the surround-screen based projection wall system. Our hypothesis was that the degree of objective psychophysiological response as well as subjective craving for cigarette smoking would be reduced after repeated exposure to VR cue provided by projection wall system.

\section{METHODS}

\section{Participants}

Ten subjects (nine male and one female) with moderate ni- cotine dependence [as assessed by the Fagerström Test for Nicotine Dependence (FTND $)^{24}$ ] who smoked cigarettes daily participated in a 4-week (one session per week) virtual cue exposure therapy. These subjects were recruited via smoking quit clinic in the SMG-SNU Boramae Medical Center and did not have nicotine replacement treatment as well as any other medications during the period of study. Simple counseling such as management of nicotine withdrawal symptoms was provided to the subjects during the study. Demographic and clinical characteristics of the subjects are summarized in Table 1. Exclusion criteria of the present study included a known history of alcohol or drug abuse/dependence other than nicotine, neurological disease or brain injury, evidence of medical illness that could manifest psychiatric symptoms, and any psychiatric disorders confirmed with the Structured Clinical Interview for DSM-IV (SCID-IV). ${ }^{25}$ This study was conducted in accordance with the Declaration of Helsinki. The Institutional Review Board of SMG-SNU Boramae Medical Center, Seoul, Korea approved this study.

\begin{abstract}
Measures
After obtaining informed consent, the researchers administered questionnaires and rating scales, including a smoking history, FTND, and Questionnaire of Smoking Urges (QSU). ${ }^{26}$ During the VR exposure session, subjects were also asked to check a self-rating Visual Analog Scale (VAS) to record their level of nicotine craving (0-100) at the moment of each cue. In order to determine whether participants smoked or not, expiratory carbon monoxide (CO) levels were obtained from each subject. The full 30-item version of the Simulator Sickness Questionnaire (SSQ) was applied to provide an index of nausea, and oculomotor- and disorientation-related symptom experiences. ${ }^{27}$ In addition, abstinences at week 4 and at week 12 of follow-up visit were determined by self-report and verified by an expiratory CO levels $\leq 6$.

Psychophysiological response data [electromyography (EMG), SC, and HR] were acquired during each VR exposure session
\end{abstract}

Table 1. Demographic and clinical characteristics

\begin{tabular}{|c|c|c|c|c|}
\hline \multirow{2}{*}{ Variables } & \multicolumn{2}{|c|}{ 4-session treatment } & \multicolumn{2}{|c|}{ Statistic } \\
\hline & Pre- & Post- & $\mathrm{F}$ & $\mathrm{p}$ \\
\hline Age & $26.20(5.14)$ & & & \\
\hline Sex & $\mathrm{M}=9, \mathrm{~F}=1$ & & & \\
\hline Duration of smoking (yrs) & $4.57(2.23)$ & & & \\
\hline Daily smoking count & $14.29(6.08)$ & $4.36(6.39)$ & 22.60 & $<0.01^{*}$ \\
\hline FTND & $5.71(1.25)$ & $0.86(1.46)$ & 204.00 & $<0.01^{*}$ \\
\hline QSU & $125.14(21.54)$ & $91.14(35.13)$ & 9.49 & $0.02^{*}$ \\
\hline Expiratory CO level (ppm) & $12.50(2.17)$ & $2.50(2.92)$ & 53.49 & $<0.01^{*}$ \\
\hline
\end{tabular}

${ }^{*} \mathrm{p}<0.05$. FTND: Fagerström Test for Nicotine Dependence, QSU: Questionnaire of Smoking Urges, CO: carbon monoxide 
using the multi-modality encoding system of the biofeedback unit and BioGraph Infiniti ver. 4.0 software (ProComp; Thought technology Ltd., Quebec, Canada). HR electrodes were attached to the middle finger of the nondominant hand, and SC electrodes were attached to the index and fourth fingers of the nondominant hand. EMG electrodes were attached to the frontalis muscle. EMG data were collected at $2,048 \mathrm{~Hz}$, while SC and HR data were collected at $256 \mathrm{~Hz}$.

\section{VR environments}

As shown in Figure 1, the 3-dimensional VR environments were implemented on a high-speed PC computer and visual stimuli were delivered to three surround screens (80 inches) through the projection of three LCD digital projectors (LX400; Christie Digital Systems, CA, USA). Motion recognition camera (HVR-2000, Hyvision, Korea) was used to detect the participant's motion and the mouse was interfaced with the computer to check the degree of subjective craving.

The authors developed virtual bar environments based on the results of survey about the frequent smoking situations. Smoking cues within virtual bar consisted of two environments. First environment provided music and several objects related to cigarette smoking, such as advertising posters, movie, burning cigarettes, alcoholic beverages, and avatars smoking cigarettes and drinking alcohols. Second environment presented a setting in which the participants engaged in some kinds of dialogues with an avatar friend, associated with social situations in the family and work places. In addition, two kinds of neutral cue environments (park or aquarium) devoid of smoking cues were used.

\section{Design and procedures}

The experimental procedure is displayed in Figure 2. After completion of baseline measurements, participants were given four serial VR cue exposure environments in the surround-screen projection room with real-time psychophysiological response monitoring. In the first environment, participants could choose a neutral park or aquarium cue and observed the neutral cue for 3 minutes, during which they felt relaxed. Environment 2 (duration, 7 minutes) provided several objects related to cigarette smoking, and environment 3 (duration, 11 minutes) consisted of various dialogues with an avatar friend, which were associated with social situations in the family and work places. In the last environment (duration, 3 minutes), participants were given a similar neutral cue as in the environment 1. After each environment ended, participants were asked to fill out an onscreen VAS to check the severity of nicotine craving using a mouse button. A VR cue exposure therapy was conducted once a week for 4 weeks. After the end of each session, the researchers administered FTND, QSU, and expiratory CO level to each subject.

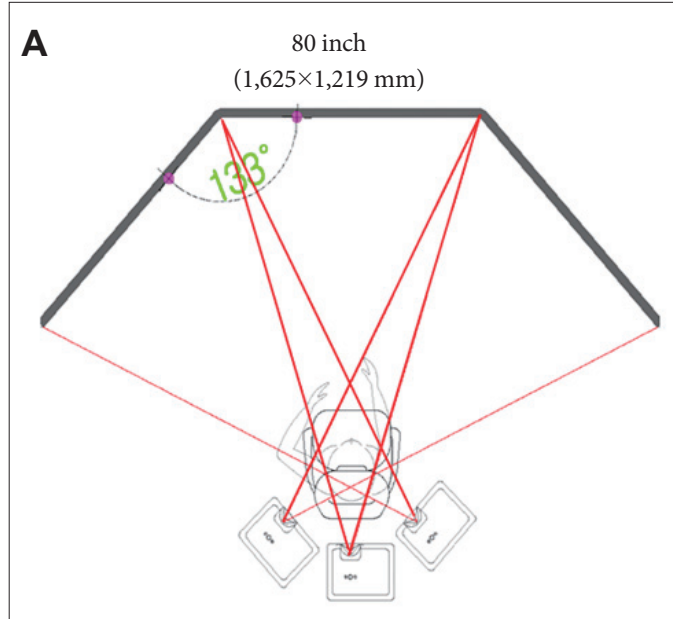

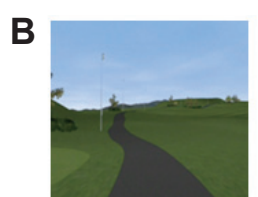

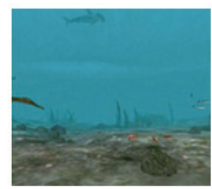

Neutral cue

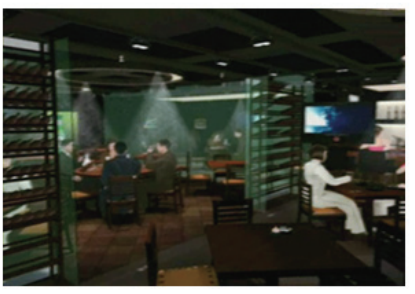

Virtual bar
Figure 1. Illustration of projection wall system (A) and stimuli used in this study (B). Projection wall system was comprised of three surround screens and three LCD digital projectors.

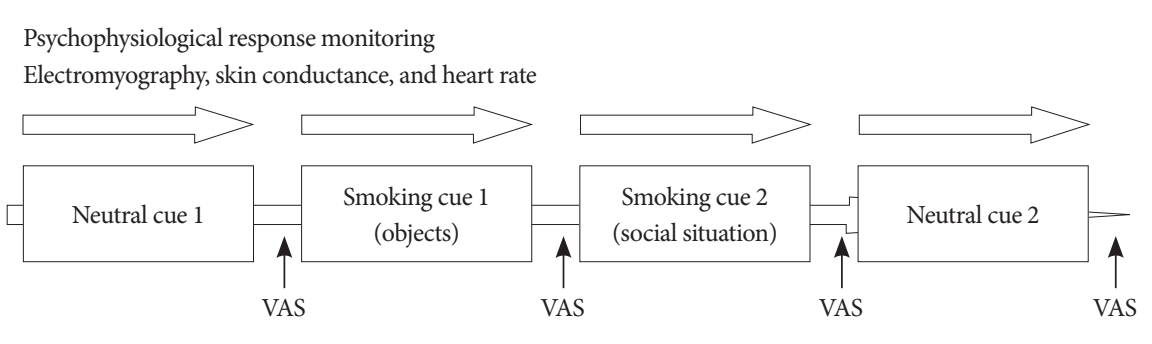

Figure 2. Study procedure during one session. VAS: Visual Analog Scale. 


\section{Statistical analysis}

A repeated measure analysis of variance (ANOVA) was used in the analysis of clinical and psychophysiological data. Statistical analysis was two-tailed and significance level was set at $\mathrm{p}<0.05$.

\section{RESULTS}

Significant decreases in the daily smoking count, FTND, QSU, and expiratory CO level of the participants were observed after a 4-week VR cue exposure therapy (Table 1). A repeated measure ANOVA revealed significant main effects on types of cue during each session $[\mathrm{F}(3,24)=22.03, \mathrm{p}<0.01]$ and session number $[\mathrm{F}(1,8)=8.62, \mathrm{p}=0.01]$ in the subjective nicotine craving using VAS during VR session over a 4-week therapy (Figure 3). Post hoc analysis confirmed that VAS scores during the exposure of object and situational cues of the first session were significantly higher than those during the exposure of neutral cues $(\mathrm{p}<0.05)$. In addition, subjective craving to nico- tine cues during the first session significantly decreased after a 4-week therapy.

With regard to the psychophysiological data, the cue reactivity patterns showed significant main effects on types of cue during each session $[\mathrm{F}(3,24)=11.07, \mathrm{p}<0.01]$ and session number $[F(1,8)=8.54, p=0.01]$ in the SC, with no significant interaction between types of cue and session number. Significant main effects on types of cue during each session $[\mathrm{F}(3,24)=$ 21.01, $\mathrm{p}<0.01]$ and session number $[\mathrm{F}(1,8)=5.96, \mathrm{p}=0.03]$ were observed in the EMG, with a significant interaction between types of cue and session number $[\mathrm{F}(3,24)=11.56, \mathrm{p}<$ 0.01]. More importantly, social situation cue elicited significantly greater cue reactivity in the SC and EMG compared to both object and neutral cues during the first session (post hoc, $\mathrm{p}<0.05$ ). In addition, these psychophysiological responses decreased after repeated exposure to VR cues. However, in the $\mathrm{HR}$, the results showed no significant main effect on types of cue during each session or session number with no significant interaction between types of cue and session number (Figure 3).

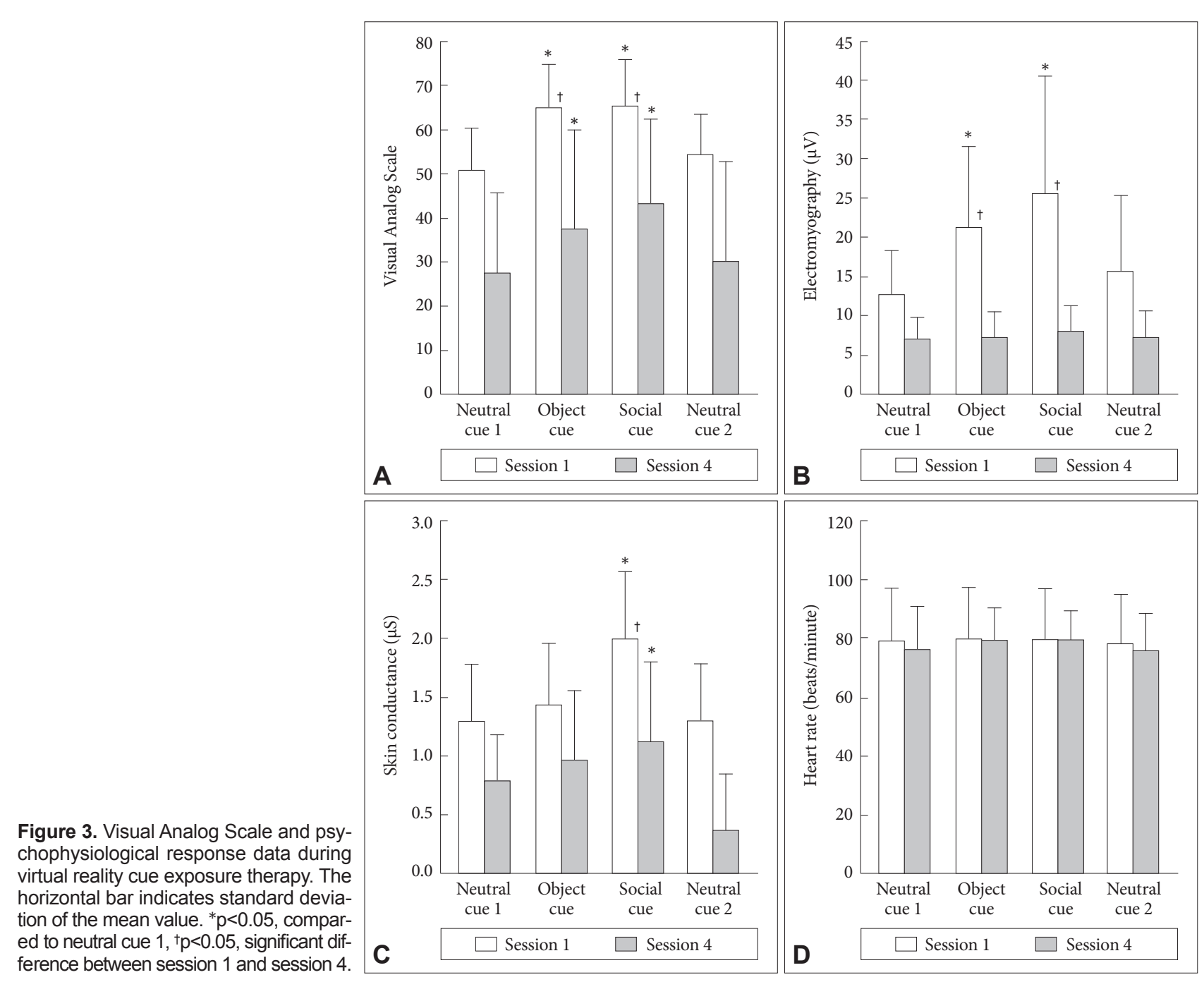


On the other hand, participants expressed low level of simulator sickness, with total score of SSQ 32.33 (SD=14.02). Continuous abstinence rates at week 4 and week 12 were $70 \%$ and $40 \%$, respectively.

\section{DISCUSSION}

In the present preliminary study, we found that VR nicotine cue with projection wall system elicited more psychophysiological response and subjective craving than did neutral cue, and this responsiveness decreased during the course of therapy. To our knowledge, this is the first report about the effect of VR cue exposure therapy using projection wall system on the psychophysiological responses in nicotine dependence.

In nicotine dependence, environmental cues associated with nicotine delivery may be important for maintaining nicotineseeking behavior. ${ }^{28,29}$ Of various environmental cues, social cues including interpersonal interaction could elicit more nicotine craving. ${ }^{5}$ In this study, greater psychophysiological responses were observed in social interaction cues relative to object cues associated with smoking and neutral cues. This suggests that social situations may be considered a main focus when treating nicotine dependence.

Because exposure to craving cues affects the psychophysiological response differently, it is necessary to measure objective physical parameter as well as subjective craving. In addition, psychophysiological monitoring seems to hold clear promises to help evaluate virtual exposure outcomes objectively and to enhance the understanding of the underlying mechanisms..$^{30}$ In this study, EMG, SC and HR were simultaneously monitored during VR cue exposure. Psychophysiological responses to social situation cue were greater than to nicotine related object and neutral cues, like the pattern of subjective nicotine craving. In particular, SC and EMG responses decreased significantly after a 4-week therapy. However, HR response during VR cue exposure was minimal, with no significant difference between the first and fourth therapy sessions. The lack of HR responsiveness might be associated with VR specific effects, such as the influences of visual attention. ${ }^{31}$ Wilhelm et al..$^{30}$ reported that VR exposure to fearful situation activated electrodermal response but not HR response, just like the results of the present study. Taken together, VR cue exposure therapy could elicit psychophysiological response as well as subjective nicotine craving. This cue reactivity decreased significantly through repeated exposure, enhancing the capability of smoking cessation. In addition, objective psychophysiological response to craving cues may be used for motivating an addicted individual's treatment. Most addicted patients would lack the insight about their problem. Through the feedback based on psychophysiological data, it may be possible to enhance the insight of the patients.

The present study introduced the projection wall system as a method of VR exposure. Losing a sense of location and orientation in a virtual world using HMD could lead to fear in the viewer and potential nausea called cybersickness. ${ }^{32,33}$ In a projection based system, disorientation and nausea might be less of an issue because the user's view is not isolated. The participants could be immersed in a VR environment, being conscious of the real world surroundings and their own body. The subjects in this study reported low level of cybersickness, suggesting that the projection wall system might be safe and provide virtual world more realistically. Cognitive behavioral therapy (CBT) has been considered to be effective in treating nicotine dependence. An important component used in CBT is a cue exposure. Based on the current results, combining CBT with VR cue exposure therapy would be more effective. In particular, cue exposure with a projection wall type VR could provide various stimuli simultaneously and more realistically, which would give participants a sense of presence or immersion in the stimulus environment.

There were several limitations in this study. First, sample size and the total number of VR therapy sessions were small: therefore, a longer therapy with larger sample would be needed. Second, only one female was included in this study. Cardiovascular reactivity in response to stressful situations could differ between men and women, ${ }^{34}$ and predict smoking outcomes differentially. ${ }^{35}$ However, after excluding one female subject, there was no significant change in the current results. In addition, subjects in this study were relatively young. Therefore, it may be difficult to generalize current results to other population. In future studies, gender differences in cue reactivity and therapy outcomes would need to be clarified and inclusion of older aged subjects would be needed. Third, this study did not employ a control group, and presentations of object-related environment and social situation environment were not counterbalanced, which could not rule out the carryover effect that craving was accumulating over the course of cue exposures. However, the current study was preliminary in nature and main focus was to determine if the degree of objective psychophysiological response as well as subjective craving for cigarette smoking would be reduced after repeated VR cue exposure provided using a projection wall system. Lastly, we could not obtain urine cotinine levels as another objective marker of nicotine smoking.

In summary, the present study found that both psychophysiological responses and subjective nicotine craving to nicotine cue exposure via projection wall VR system were greater than to neutral cues and that this enhanced cue reactivity decreased gradually over the course of therapy. These results suggest that VR cue exposure therapy combined with psychophy- 
siological response monitoring may be an alternative treatment modality for smoking cessation, although the current findings are preliminary.

\section{Acknowledgments}

We thank InWook Choi at School of Industrial \& Media Design, Handong Global University and Duke Jung at FNI Co., Ltd. for the technological support of production of virtual reality contents.

\section{REFERENCES}

1. Fagerström K. The epidemiology of smoking: health consequences and benefits of cessation. Drugs 2002;62 Suppl 2:1-9.

2. Shiffman S, Mason KM, Henningfield JE. Tobacco dependence treatments: review and prospectus. Annu Rev Public Health 1998;19:335358.

3. Conklin CA, Tiffany ST. Applying extinction research and theory to cue-exposure addiction treatments. Addiction 2002;97:155-167.

4. Saladin ME, Brady KT, Graap K, Rothbaum BO. A preliminary report on the use of virtual reality technology to elicit craving and cue reactivity in cocaine dependent individuals. Addict Behav 2006;31:1881-1894.

5. Niaura R, Abrams DB, Pedraza M, Monti PM, Rohsenow DJ. Smokers' reactions to interpersonal interaction and presentation of smoking cues. Addict Behav 1992;17:557-566.

6. Droungas A, Ehrman RN, Childress AR, O'Brien CP. Effect of smoking cues and cigarette availability on craving and smoking behavior. Addict Behav 1995;20:657-673.

7. Niaura R, Abrams D, Demuth B, Pinto R, Monti P. Responses to smoking-related stimuli and early relapse to smoking. Addict Behav 1989; 14:419-428.

8. Jerome LW, Jordan PJ, Rodericks R, Fedenczuk L. Psychophysiological arousal and craving in smokers, deprived smokers, former smokers, and non-smokers. Stud Health Technol Inform 2009;144:179-183.

9. Kang Y, Cappella JN, Strasser AA, Lerman C. The effect of smoking cues in antismoking advertisements on smoking urge and psychophysiological reactions. Nicotine Tob Res 2009;11:254-261.

10. Miranda R Jr, Rohsenow DJ, Monti PM, Tidey J, Ray L. Effects of repeated days of smoking cue exposure on urge to smoke and physiological reactivity. Addict Behav 2008;33:347-353.

11. Killen JD, Fortmann SP. Craving is associated with smoking relapse: findings from three prospective studies. Exp Clin Psychopharmacol 1997;5:137-142.

12. Marlatt GA. Cue exposure and relapse prevention in the treatment of addictive behaviors. Addict Behav 1990;15:395-399.

13. Rohsenow DJ, Niaura RS, Childress AR, Abrams DB, Monti PM. Cue reactivity in addictive behaviors: theoretical and treatment implications. Int J Addict 1990-1991;25:957-993.

14. Lee J, Lim Y, Graham SJ, Kim G, Wiederhold BK, Wiederhold MD, et al. Nicotine craving and cue exposure therapy by using virtual environments. Cyberpsychol Behav 2004;7:705-713.

15. McClernon FJ, Hiott FB, Liu J, Salley AN, Behm FM, Rose JE. Selectively reduced responses to smoking cues in amygdala following extinction-based smoking cessation: results of a preliminary functional magnetic resonance imaging study. Addict Biol 2007;12:503-512.

16. Moon J, Lee JH. Cue exposure treatment in a virtual environment to reduce nicotine craving: a functional MRI study. Cyberpsychol Behav
2009;12:43-45.

17. Emmelkamp PM, Krijn M, Hulsbosch AM, de Vries S, Schuemie MJ, van der Mast CA. Virtual reality treatment versus exposure in vivo: a comparative evaluation in acrophobia. Behav Res Ther 2002;40:509-516.

18. da Costa RT, Sardinha A, Nardi AE. Virtual reality exposure in the treatment of fear of flying. Aviat Space Environ Med 2008;79:899-903.

19. Rothbaum BO, Hodges LF, Ready D, Graap K, Alarcon RD. Virtual reality exposure therapy for Vietnam veterans with posttraumatic stress disorder. J Clin Psychiatry 2001;62:617-622.

20. Bordnick PS, Traylor A, Copp HL, Graap KM, Carter B, Ferrer M, et al. Assessing reactivity to virtual reality alcohol based cues. Addict Behav 2008;33:743-756.

21. Bordnick PS, Copp HL, Traylor A, Graap KM, Carter BL, Walton A, et al. Reactivity to cannabis cues in virtual reality environments. J Psychoactive Drugs 2009;41:105-112.

22. Bordnick PS, Graap KM, Copp HL, Brooks J, Ferrer M. Virtual reality cue reactivity assessment in cigarette smokers. Cyberpsychol Behav 2005;8:487-492.

23. Maltby N, Kirsch I, Mayers M, Allen GJ. Virtual reality exposure therapy for the treatment of fear of flying: a controlled investigation. J Consult Clin Psychol 2002;70:1112-1118.

24. Heatherton TF, Kozlowski LT, Frecker RC, Fagerström KO. The Fagerström Test for Nicotine Dependence: a revision of the Fagerström Tolerance Questionnaire. Br J Addict 1991;86:1119-1127.

25. First MB, Spitzer RL, Gibbon M, Williams JBW. Structured Clinical Interview for DSM-IV Axis I Disorder. New York: New York State Psychiatric Institute; 1996.

26. Tiffany ST, Drobes DJ. The development and initial validation of a questionnaire on smoking urges. Br J Addict 1991;86:1467-1476.

27. Kennedy RS, Lane NE, Berbaum KS, Lilienthal MG. Simulator sickness questionnaire: an enhanced method for quantifying simulator sickness. Int J Aviat Psychol 1993;3:203-220.

28. Balfour DJ, Wright AE, Benwell ME, Birrell CE. The putative role of extra-synaptic mesolimbic dopamine in the neurobiology of nicotine dependence. Behav Brain Res 2000;113:73-83.

29. Donny EC, Caggiula AR, Rowell PP, Gharib MA, Maldovan V, Booth S, et al. Nicotine self-administration in rats: estrous cycle effects, sex differences and nicotinic receptor binding. Psychopharmacology (Berl) 2000; 151:392-405.

30. Wilhelm FH, Pfaltz MC, Gross JJ, Mauss IB, Kim SI, Wiederhold BK. Mechanisms of virtual reality exposure therapy: the role of the behavioral activation and behavioral inhibition systems. Appl Psychophysiol Biofeedback 2005;30:271-284.

31. de Carvalho MR, Freire RC, Nardi AE. Virtual reality as a mechanism for exposure therapy. World J Biol Psychiatry 2010;11:220-230.

32. Kiryu T, So RH. Sensation of presence and cybersickness in applications of virtual reality for advanced rehabilitation. J Neuroeng Rehabil 2007; 4:34.

33. Viaud-Delmon I, Warusfel O, Seguelas A, Rio E, Jouvent R. High sensitivity to multisensory conflicts in agoraphobia exhibited by virtual reality. Eur Psychiatry 2006;21:501-508.

34. Matthews KA, Davis MC, Stoney CM, Owens JF, Caggiula AR. Does the gender relevance of the stressor influence sex differences in psychophysiological responses? Health Psychol 1991;10:112-120.

35. Swan GE, Ward MM, Jack LM, Javitz HS. Cardiovascular reactivity as a predictor of relapse in male and female smokers. Health Psychol 1993; 12:451-458. 